\title{
Progressive disseminated histoplasmosis presenting with cachexia and hypercalcemia
}

This article was published in the following Dove Press journal:

International Journal of General Medicine

26 February 2013

Number of times this article has been viewed

Faisal A Khasawneh'

Subhan Ahmed ${ }^{2}$

Ruba A Halloush ${ }^{3}$

'Section of Infectious Diseases, Department of Internal Medicine, Texas Tech University Health Sciences Center, Amarillo, TX, ${ }^{2}$ Section of Nephrology, Department of Internal Medicine, University of Oklahoma, Tulsa, OK, ${ }^{3}$ Amarillo Pathology Group, Amarillo, TX, USA
Correspondence: Faisal A Khasawneh Section of Infectious Diseases, Department of Internal Medicine, Texas Tech University Health Sciences Center, 1400 S Coulter Street, Amarillo, TX 79106, USA

Tel +l 8063545480

Fax +l 8063545765

Email faisal.khasawneh@ttuhsc.edu

\begin{abstract}
Histoplasmosis is a common endemic mycosis. The majority of infections involving this dimorphic fungus are asymptomatic. Manifestations in symptomatic patients are diverse, ranging from flu-like illness to a more serious disseminated disease. We present here a case of chronic disseminated histoplasmosis mimicking a metastatic cancer. We reviewed the literature for cases of disseminated histoplasmosis presenting with hypercalcemia, focusing particularly on clinical presentation, risk factors predisposing for fungal infection, and outcome. We report a case of a 65-year-old diabetic male who presented with unexplained weight loss and hypercalcemia. Multiple brain space-occupying lesions and bilateral adrenal enlargement were evident on imaging studies. Biopsies showed caseating granulomas with budding yeast, consistent with histoplasmosis. The patient's symptoms resolved after liposomal amphotericin B and itraconazole therapy. Granulomatous diseases, including fungal infections, should be considered alongside malignancies, in patients with similar presentation.
\end{abstract}

Keywords: disseminated histoplasmosis, hypercalcemia

\section{Introduction}

Histoplasmosis is a fungal infection caused by the dimorphic fungus, Histoplasma capsulatum. It is the most common endemic mycosis in the United States. ${ }^{1}$ It is most prevalent around the valleys of the Mississippi and Ohio rivers. ${ }^{2}$ In endemic areas, $50 \%-80 \%$ of people have evidence of previous exposure to histoplasma. ${ }^{3}$ The fungus grows as a mold in the soil and when its microconidia are inhaled, causes infection and grows as a yeast in the host tissues. Most infected people remain asymptomatic or complain of a self-limiting flu-like illness. Up to $25 \%$ of people infected with human immunodeficiency virus will develop disseminated histoplasmosis, with considerable morbidity and mortality. ${ }^{3}$ Infection outside endemic areas and atypical presentations represent a diagnostic challenge. We present a case of progressive disseminated histoplasmosis manifesting as a wasting syndrome with hypercalcemia, mimicking a metastatic cancer.

\section{Case report}

A 65-year-old, type 2 diabetic man presented with a 2-month history of constipation, polyuria, and unexplained weight loss of $54 \mathrm{lb}$. There was no fever or chills and no respiratory symptoms. The patient was a lifelong smoker. He had lived in West Pennsylvania until 13 years earlier, when he had moved to the Texas Panhandle area where he presented with the above complaints. On physical examination, the patient was afebrile. He was confused and cachectic, without neck masses, lymphadenopathy, or organomegaly. 


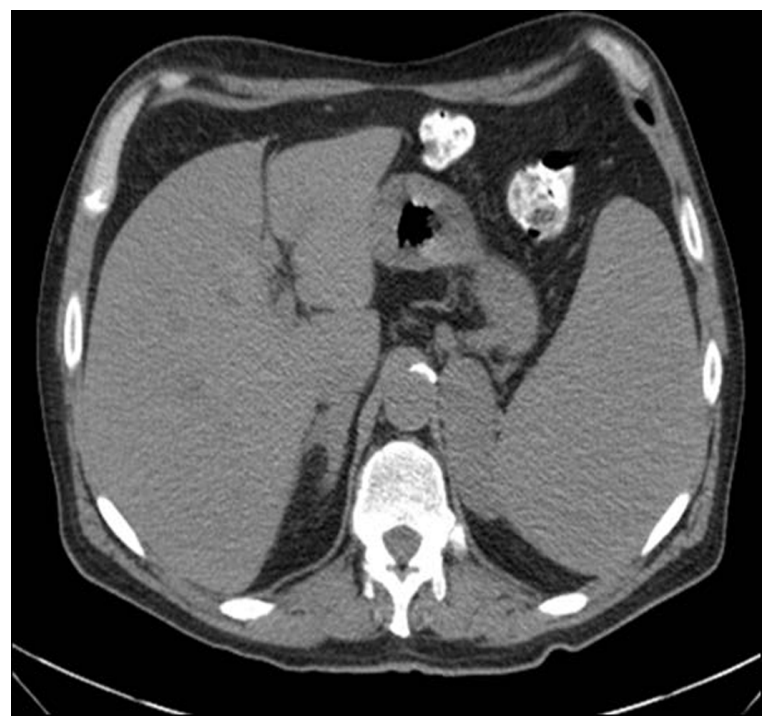

Figure I Computed tomography of the abdomen showing enlarged adrenal glands, the left gland measured $6.8 \times 6.7 \times 2.7 \mathrm{~cm}$.

The patient did not have skin rashes or mucosal ulcers. There were no heart murmurs and no adventitial lung sounds.

Laboratory test results revealed a hemoglobin of 10.6 (normal range 12-16) $\mathrm{g} / \mathrm{dL}$ and a white blood cell count of $3.5 \times 10^{9} \mathrm{cells} / \mathrm{L}$ (normal range $4.0-10.6 \times 10^{9} \mathrm{cells} / \mathrm{L}$ ). Biochemistry tests showed a creatinine of 3.2 (normal range $0.5-1.4) \mathrm{mg} / \mathrm{dL}$, serum calcium of 12.4 (normal range 8.4-10.3) $\mathrm{mg} / \mathrm{dL}$, and albumin of 3.5 (normal range 3.7-5.1) $\mathrm{g} / \mathrm{dL}$. The patient's parathyroid hormone level was low at 6 (normal range 11.0-54.0) $\mathrm{pg} / \mathrm{mL}$ and serum protein electrophoresis showed a normal pattern.
Body computed tomography showed bilateral adrenal enlargement and a mass lesion at the base of the tongue (Figure 1). Magnetic resonance imaging of the brain showed three left-sided brain lesions (Figure 2). Biopsies of the tongue lesion and the left adrenal gland showed necrotizing granulomas containing budding yeast forms, consistent with histoplasmosis (Figures 3-5). Biopsy tissue cultures were negative. The patient was diagnosed with chronic progressive disseminated histoplasmosis. Histoplasmosis is rarely diagnosed in the Texas Panhandle area and we were unable to tell whether his presentation represented a reactivation of an old infection or progression of a newly acquired infection. No other cases of histoplasmosis were diagnosed in our hospital during that time frame.

After adequate intravenous hydration, the patient's kidney function tests and serum calcium level reverted to normal. The patient received a 4-week course of liposomal amphotericin B and was subsequently started on itraconazole. The patient's symptoms resolved and he is being followed on regular basis.

\section{Discussion}

Dissemination of $H$. capsulatum is common in the early stages of this fungal infection. ${ }^{1}$ Symptomatic acute dissemination develops in immunocompromised patients. ${ }^{2}$ They present with a febrile illness that can be complicated by severe sepsis, acute respiratory distress syndrome, and disseminated intravascular coagulopathy. On the other hand, chronic progressive disseminated histoplasmosis is

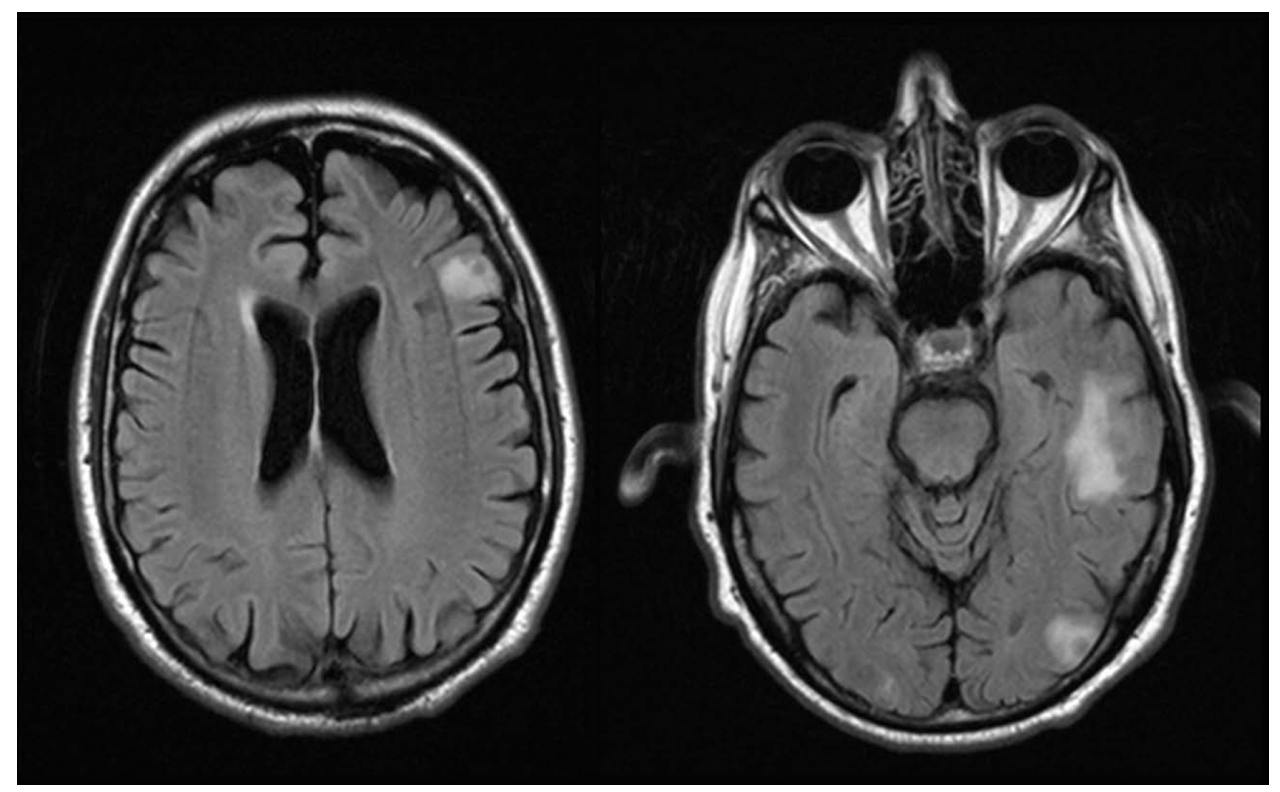

Figure 2 Brain magnetic resonance imaging (flare) showing three lesions in the left frontal, temporal, and occipital lobes. 


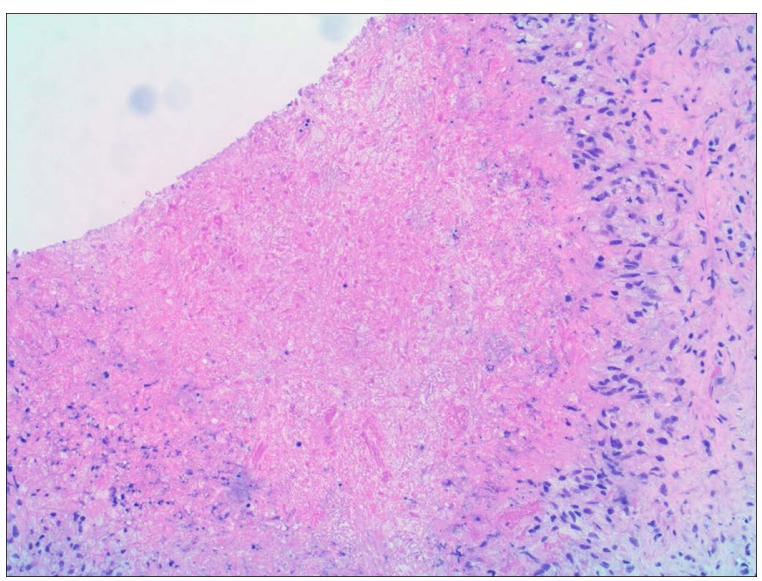

Figure 3 Adrenal needle biopsy section stained with hematoxylin and eosin showing necrotizing granuloma (original magnification, 10x).

typically reported in middle-aged and elderly men who are not immunosuppressed. ${ }^{3}$ They present with a wasting syndrome, long-standing fever, and night sweats. The infection may involve multiple organ systems, including the gastrointestinal tract (with resulting ulceration), adrenal glands (precipitating adrenal insufficiency), the reticuloendothelial system (causing hepatosplenomegaly), bone marrow (leading to pancytopenia), the central nervous system, and the lungs. On rare occasions, progressive disseminated histoplasmosis has been associated with hypercalcemia, and this is attributed to increased 1, 25 dihydroxyvitamin $\mathrm{D}$ production from the fungal granulomas. ${ }^{4-10}$

A Medline search (January, 1946 to November, 2012) identified seven reported cases of disseminated histoplasmosis presenting with hypercalcemia. The clinical presentation, risk factors that predisposed to histoplasmosis, and patient outcomes are reported (Table 1). Upon reviewing

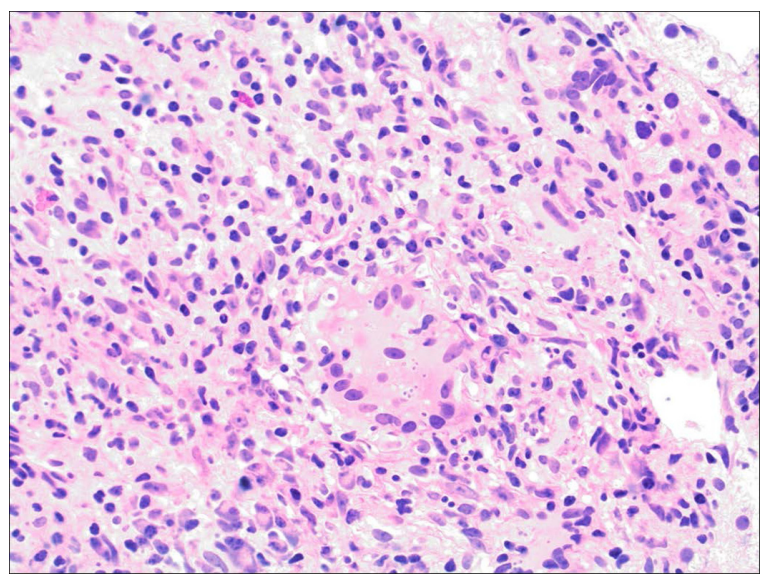

Figure 4 Adrenal needle biopsy section stained with hematoxylin and eosin showing granulomatous inflammation and a multinucleated giant cell containing small yeast surrounded by halos representing fungal capsules (original magnification, 20x).

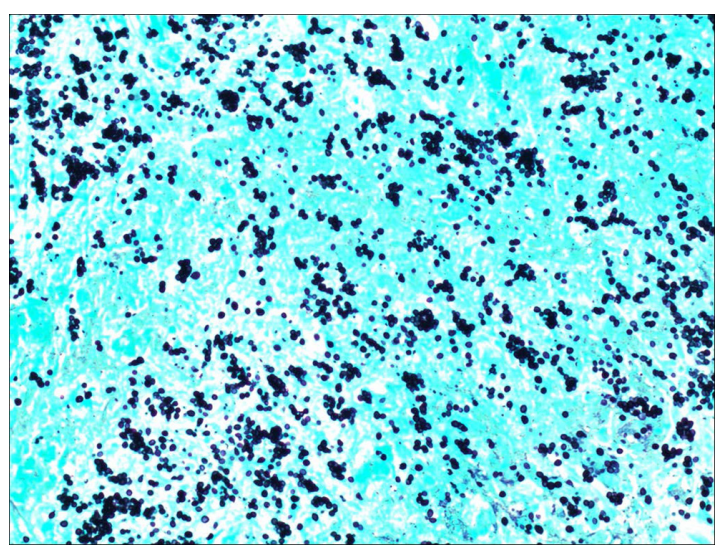

Figure 5 Adrenal needle biopsy section stained with Gomori methenamine silver showing small oval budding yeast typical of Histoplasma capsulatum (original magnification, 40×).

the cases and in comparison with the case at hand, the following features were noted. Like our patient, most patients were middle-aged and elderly men with a limited degree of immunosuppression. Presenting symptoms varied, with pulmonary complaints in two cases, gastrointestinal symptoms in two, wasting syndrome in three, including ours, and musculoskeletal complaints in another. Hypercalcemia was symptomatic in some cases and an asymptomatic laboratory abnormality in others. In all cases, the diagnosis was difficult to make, and in three cases was established post mortem. Mortality was high, with $37.5 \%$ of the patients dying. Two of the three patients who died, ie, the first and second cases, did not receive antifungal therapy, and treatment was delayed in the third patient who died, ie, the fourth case.

Diagnosis of histoplasmosis relies on a multifaceted approach. ${ }^{11}$ Histopathological examination, cultures, antigen and antibody detection, and molecular methods are commonly used in different combinations to establish the diagnosis. A recent multicenter study evaluated the above-mentioned tests in the diagnosis of disseminated histoplasmosis and reported their corresponding sensitivities, ie, $74 \%$ for cultures, $76 \%$ for histopathology, $92 \%$ for antigen detection in urine and/or serum using a third-generation enzyme immunoassay, and $75 \%$ for antibody detection combining immunodiffusion and complement fixation assays. ${ }^{11}$ Use of molecular methods in the diagnosis of histoplasmosis has been reported, but remains uncertain and is awaiting further study. ${ }^{12}$

The high sensitivity of antigen detection is plagued by significant cross-reactivity with other fungal antigens. Crossreaction occurs in $90 \%$ of patients with blastomycosis and in $60 \%$ of patients with coccidioidomycosis. ${ }^{13}$ Testing both urine and serum yields better sensitivity. Furthermore, testing cerebrospinal fluid and bronchoalveolar lavage fluid might 
Table I Reported cases of progressive disseminated histoplasmosis presenting with hypercalcemia

\begin{tabular}{llll}
\hline Age/gender & Risk factors & Presenting symptoms & Reference \\
\hline 56 years, $M$ & Splenectomy and & Fever, malaise, nausea, & 4 \\
62 years, $M$ & steroid therapy & vomiting, confusion weight loss & Malaise, anorexia, weight loss \\
10 months, M & DM & Failure to thrive & 5 \\
46 years, $M$ & Immature immunity & Fever, diarrhea, papular & 7 \\
47 years, $M$ & Malnutrition due & skin rash \\
40 years, F & to chronic diarrhea & Right hand tenosynovitis & 8 \\
& None & Fever, cough, nodular & 9 \\
61 years, $M$ & RA on infliximab, & pulmonary infiltate, & \\
65 years, $M$ & methotrexate, & lymphadenopathy & 10 \\
\hline
\end{tabular}

Abbreviations: DM, diabetes mellitus; F, female; M, male; RA, rheumatoid arthritis.

improve sensitivity in diagnosing central nervous system and pulmonary infections..$^{14}$ It is worth mentioning that failure to detect histoplasma antigens does not rule out the diagnosis, and repeating the test in patients with progressive illness should be considered. ${ }^{3}$

Severity of illness dictates antifungal treatment options and duration of therapy. For moderate to severe infection, liposomal amphotericin B for 1-2 weeks followed by a 12-month course of itraconazole is recommended. ${ }^{15}$ For milder cases, itraconazole for one year is indicated. Itraconazole blood levels should be measured to ensure adequate drug exposure. For histoplasmosis of the central nervous system, liposomal amphotericin B for 4-6 weeks followed by itraconazole for at least one year and until cerebrospinal fluid abnormalities and antigenemia or antigenuria resolve is recommended..$^{15}$ Antigen levels in serum or urine should be measured during therapy for progressive disseminated histoplasmosis and central nervous system infection, and for 12 months afterwards. Ten percent to $15 \%$ of patients experience a relapse. ${ }^{16}$ Diagnosis and treatment in this group of patients follows the above outlined principles, but also includes long-term itraconazole maintenance therapy.

Our work has some limitations, not the least of which is the fact that it is a single case report. Furthermore, the diagnosis was based on a compatible clinical presentation and histopathological examination that could not be confirmed by culture, serology, or antigen detection. Further research is needed to develop readily available tests, probably molecular diagnostic methods, with higher sensitivity and specificity to diagnosis histoplasmosis as well as other mycosis. Efforts to develop safer and more effective antifungal drugs would also be worthwhile.

\section{Conclusion}

Progressive disseminated histoplasmosis is a fungal infection with diverse presentations. Acute disseminated infection presents with a sepsis syndrome, whereas chronic dissemination presents as a wasting syndrome. There are rare manifestations of this mycosis that could delay diagnosis and compromise outcome. This infection is fatal if not treated. We reported here a case of chronic disseminated histoplasmosis presenting with multiple mass lesions, weight loss, and hypercalcemia, mimicking metastatic cancer. The patient was treated successfully with appropriate antifungal therapy. In addition to malignancy, granulomatous disease, including fungal infection, should be considered in patients with similar presentation.

\section{Disclosure}

The authors report no conflicts of interest in this work.

\section{References}

1. Kauffman CA. Histoplasmosis. Clin Chest Med. 2009;30(2):217-225, v.

2. Knox KS, Hage CA. Histoplasmosis. Proc Am Thorac Soc. 2010;7(3): 169-172.

3. Assi MA, Sandid MS, Baddour LM, Roberts GD, Walker RC. Systemic histoplasmosis: a 15-year retrospective institutional review of 111 patients. Medicine (Baltimore). 2007;86(3):162-169.

4. Walker JV, Baran D, Yakub N, Freeman RB. Histoplasmosis with hypercalcemia, renal failure, and papillary necrosis. Confusion with sarcoidosis. JAMA. 1977;237(13):1350-1352.

5. Murray JJ, Heim CR. Hypercalcemia in disseminated histoplasmosis. Aggravation by vitamin D. Am J Med. 1985;78(5):881-884.

6. Steele CJ, Kleiman MB. Disseminated histoplasmosis, hypercalcemia and failure to thrive. Pediatr Infect Dis J. 1994;13(5):421-422.

7. Liu JW, Huang TC, Lu YC, et al. Acute disseminated histoplasmosis complicated with hypercalcaemia. J Infect. 1999;39(1):88-90.

8. Liang KV, Ryu JH, Matteson EL. Histoplasmosis with tenosynovitis of the hand and hypercalcemia mimicking sarcoidosis. J Clin Rheumatol. 2004;10(3):138-142. 
9. Kamili QA, Menter A. Atypical presentation of histoplasmosis in a patient with psoriasis and psoriatic arthritis on infliximab therapy J Drugs Dermatol. 2010;9(1):57-60.

10. Chalhoub E, Elhomsy G, Brake M. Hypercalcemia in histoplasmosis aggravated with antifungal treatment. J Med Liban. 2012;60(3): $165-168$.

11. Hage CA, Ribes JA, Wengenack NL, et al. A multicenter evaluation of tests for diagnosis of histoplasmosis. Clin Infect Dis. 2011;53(5): $448-454$.

12. Bracca A, Tosello ME, Girardini JE, Amigot SL, Gomez C, Serra E. Molecular detection of Histoplasma capsulatum var. capsulatum in human clinical samples. J Clin Microbiol. 2003;41(4):1753-1755.
13. Connolly PA, Durkin MM, Lemonte AM, Hackett EJ, Wheat LJ. Detection of histoplasma antigen by a quantitative enzyme immunoassay. Clin Vaccine Immunol. 2007;14(12):1587-1591.

14. Hage CA, Davis TE, Fuller D, et al. Diagnosis of histoplasmosis by antigen detection in BAL fluid. Chest. 2010;137(3):623-628.

15. Wheat LJ, Freifeld AG, Kleiman MB, et al. Clinical practice guidelines for the management of patients with histoplasmosis: 2007 update by the Infectious Diseases Society of America. Clin Infect Dis. 2007;45(7): $807-825$.

16. Wheat J, Hafner R, Korzun AH, et al. Itraconazole treatment of disseminated histoplasmosis in patients with the acquired immunodeficiency syndrome. AIDS Clinical Trial Group. Am J Med. 1995;98(4):336-342.
International Journal of General Medicine

\section{Publish your work in this journal}

The International Journal of General Medicine is an international, peer-reviewed open-access journal that focuses on general and internal medicine, pathogenesis, epidemiology, diagnosis, monitoring and treatment protocols. The journal is characterized by the rapid reporting of reviews, original research and clinical studies across all disease areas.

\section{Dovepress}

A key focus is the elucidation of disease processes and management protocols resulting in improved outcomes for the patient.The manuscript management system is completely online and includes a very quick and fair peer-review system. Visit http://www.dovepress.com/ testimonials.php to read real quotes from published authors.

Submit your manuscript here: http://www.dovepress.com/international-journal-of-general-medicine-journal 